\title{
La psicomotricidad como una herramienta del juego terapéutico implementada en un centro de estimulación multisensorial
}

\section{Psychomotricity as a therapeutic game tool implemented in a multisensory stimulation center}

\author{
APAN-ARAUJO, Karla Cecilia†*, SORIANO-PORRAS, Dulce Maria, MARTÍNEZ-TÉLLEZ, \\ Rubelia Isaura y ROMERO-RODRÍGUEZ, Gabriel
}

Universidad Politécnica de Amozoc, México.

ID $1^{\mathrm{er}}$ Autor: Karla Cecilia, Apan-Araujo / ORC ID: 0000-0003-3373-226X, Researcher ID Thomson: E-8338-2018, CVU CONACYT ID: 896703

ID $1^{\mathrm{er}}$ Coautor: Dulce María, Soriano-Porras / ORC ID: 0000-0001-7398-0693, Researcher ID Thomson: E-8233-2018, arXiv ID Author: 8GO6IR-3HFFHH

ID $2^{\text {do }}$ Coautor: Rubelia Isaura, Martínez-Téllez / ORC ID: 0000-0003-0164-066X, Researcher ID Thomson: E-82292018, arXiv ID Author: GYNFU8-XHIM03

ID $3^{\text {er }}$ Coautor: Gabriel, Romero-Rodríguez / ORC ID: 0000-0002-5124-3016, Researcher ID Thomson: Y-2756-2018, CVU CONACYT ID: 299737

DOI: $10.35429 / J B E .2020 .11 .4 .11 .16$ Recibido 15 de Enero, 2020; Aceptado 30 de Junio, 2020

\section{Resumen}

La presente investigación muestra la manera de trabajar la psicomotricidad como una herramienta del juego terapéutico implementada en un Centro de Estimulación Multisensorial (CEMS) el cual se encuentra en la Universidad Politécnica de Amozoc, Puebla, México. Los objetivos son: a) Determinar el nivel de desarrollo psicomotor en preescolares, b) Desarrollar un plan de estrategias lúdicas que contribuyen al desarrollo psicomotor, c) Evaluar el desarrollo psicomotor de los niños una vez implementadas las actividades. Para ello se diseñaron actividades educativas con niños de tercer año de preescolar, en donde previamente se realizó una valoración a un grupo de niños utilizando un test específico para determinar su nivel de psicomotricidad de acuerdo a su desarrollo, los cuales fueron referidos por sus maestras. Posterior a ello, se seleccionó la población a trabajar y se establecieron una serie de actividades apoyadas por medio del juego terapéutico, de tal forma que potencializarán su desarrollo psicomotor. Por último, la contribución de dicha investigación es demostrar el impacto que se obtiene en el desarrollo psicomotor por medio del juego terapéutico en niños de nivel preescolar.

Desarrollo psicomotor, Juego terapéutico, Centro de estimulación multisensorial

\begin{abstract}
The present investigation shows the way to work the psychomotricity as a tool of the therapeutic game implemented in a Multisensory Stimulation Center (CEMS) which is located in the Universidad Politécnica de Amozoc, Puebla, Mexico. The objectives are: a) Determine the level of psychomotor development in preschoolers, b) Develop a plan of playful strategies that contribute to psychomotor development, c) Evaluate the psychomotor development of children once the activities have been implemented. For this, educational activities were designed with third year preschool children, where previously a group of children was assessed using a specific test to determine their level of psychomotricity according to their development, which were reported by their teachers. Subsequently, the population to work was selected and a series of activities supported by means of therapeutic play were established, in such a way that they potentiated their psychomotor development. Finally, the contribution of this research is to demonstrate the impact that is obtained in psychomotor development through therapeutic play in preschool children.
\end{abstract}

Psychomotor development, Therapeutic play, Multisensory stimulation center

\footnotetext{
*Correspondencia al Autor (Correo Electrónico: karla.apan@upamozoc.edu.mx)

$\dagger$ Investigador contribuyendo como primer autor.
} 


\section{Introducción}

La psicomotricidad en la etapa preescolar es un elemento fundamental dentro del desarrollo en las habilidades tanto físicas como cognitivas en los niños. El juego es una necesidad fundamental y una herramienta que permite al ser humano conocerse a sí mismo y al mundo al que pertenece. Dicho juego es algo inherente a la propia naturaleza infantil, lo que le permite conocerse a sí mismo, ser creativo, participativo, expresarse, tener amigos, compartir, enfrentarse a situaciones que debe resolver, etc.; por lo que lo convierte en una necesidad espontánea que se irá enlazando con el desarrollo integral del niño. Además, existe una relación inherente entre el juego y el desarrollo del niño; ya que ambos tienen elementos tanto psicomotores, cognitivos, sensoriomotores, intelectuales, sociales y emocionales.

En el caso de los sentidos, estos permiten recibir información del exterior, analizarla e interpretarla con cada de ellos; debido a las conexiones nerviosas con las que cuenta nuestro cerebro. Es por ello, la importancia del uso de un CEMS ya que esté proporciona información, la cual es recibida por medio de los diferentes canales sensoriales lo que permite interpretar e integrar estos estímulos por medio de nuestros sentidos, para así fortalecer aún más nuestras experiencias sensoriales y nuestro conocimiento del medio que nos rodea.

Por lo tanto, con el apoyo de un CEMS y actividades específicas se generan estímulos por medio del juego terapéutico donde los alumnos de preescolar por medio de sus sentidos interiorizan dicha información, la cual se ve reflejada en su desarrollo psicomotor más adelante.

\section{Marco Teórico}

A continuación, se describen brevemente algunos artículos sobre la psicomotricidad, el juego terapéutico y el CEMS.
El contenido de la Psicomotricidad. Reflexiones para la delimitación de su ámbito teórico y práctico. Este artículo menciona la encrucijada de la psicomotricidad, al observar la ubicación de la psicomotricidad, tanto desde el punto de vista de su ámbito de intervención, como desde el de su fundamentación y su desempeño profesional, nos damos cuenta de que sus objetivos y posibilidades se encuentran en una encrucijada situada entre la educación, la sanidad, la atención social y la prevención. Dicho de otro modo: el campo de trabajo se extiende a esos ámbitos, sin que se pierda una visión de identidad de planteamientos teóricos y prácticos. El espacio epistemológico de la psicomotricidad abarca, pues, las ciencias de la salud, de la educación, del movimiento, así como las técnicas de intervención; y el espacio profesional del psicomotricista se encuentra próximo (según el campo en que se desarrolle) al del fisioterapeuta en el área de la salud, al del profesor de educación física en el área de la educación, al del terapeuta ocupacional o ergoterapeuta en el área de la atención social, y al del estimulador en el área de la prevención. (Berruezo, 2008).

La estimulación psicomotriz temprana: Los efectos terapéuticos del movimiento. Este artículo aborda el cómo puede deducirse de la exposición previa sobre los procesos de desarrollo psicomotor, los primeros años de vida constituyen una etapa de la existencia especialmente crítica ya que en ella se van a configurar las habilidades perceptivas, motrices, cognitivas, lingüísticas, afectivas y sociales que posibilitarán una equilibrada interacción con el mundo circundante. Desde diversos ámbitos de Atención TempranaSanidad, Servicios Sociales y Educación; se ofrecen un conjunto de acciones que facilitan la maduración global adecuada del niño, su máximo nivel de desarrollo personal y de integración social. En este proceso, además del diagnóstico precoz de factores de riesgo, se enfatizan los procesos optimizadores de dicho desarrollo y la adopción de medidas preventivas, rentables a nivel social, económico, y personal. 
Muchas alteraciones de desarrollo pueden prevenirse si son tratadas a tiempo, promoviendo con ello el bienestar social de las familias y el de la sociedad en su conjunto- ya que factores como la dedicación afectiva, la estabilidad de las relaciones familiares, la suficiencia económica, la estabilidad laboral, la participación en redes sociales y la coherencia de los estilos educativos, son fundamentales en el desarrollo infantil. (Ibáñez, 2004).

La psicomotricidad pertenece al ámbito del desarrollo psicológico del ser humano con relación al mundo que lo rodea. Este artículo menciona que el movimiento tiene una gran importancia en el desarrollo integral de la infancia, por su implicación en la estructuración de la personalidad, el desarrollo del proceso comunicativo, expresión y relación con objetos, así como la creación de una relación con otras personas del entorno. Tiene una gran influencia en la adquisición de autonomía personal y de estructuración cognitiva. La psicomotricidad implica el desarrollo de aspectos socioafectivos, motores, psicomotores e intelectuales que será lo que definirá el progreso global del niño a través de la interacción de sus propias vivencias corporales que facilitan el desarrollo de las capacidades sensorio-motrices, la percepción, la comunicación y la expresión mediante la interacción de su cuerpo con el medio ambiente. (Mas, 2013).

¿Qué es una sala de estimulación multisensorial? Este artículo establece que se trata de un espacio habilitado para que los alumnos con algún tipo de deficiencia puedan despertar los sentidos e interactuar con el medio, dentro de un entorno favorecedor. Se trabaja de una manera distinta al aula, con estímulos agradables, en un ambiente tranquilo y relajado, que permite acercarnos a los alumnos de una forma muy personal e íntima, actuando de vehículo favorecedor de una estimulación sensorial que nos deja interactuar y controlar el momento, despertando las sensaciones a través de sus propias experiencias.

El espacio multisensorial conjuga la aproximación curativa y no directiva con la aportación de estimulaciones sensoriales dentro de un entorno específico utilizado para desencadenar una relación.
El objetivo propuesto es dar curso libre a la experiencia sensorial, de buscar la satisfacción, el placer y el descanso, de respetar la motivación y el ritmo de la persona. En una dimensión interior y personal, nos ayuda al reencuentro de las sensaciones más intensas y a expresar emociones contenidas. (Carbajo, 2014)

\section{Metodología}

La propuesta del trabajo práctico se refiere a la aplicación de estrategias lúdicas convertidas en actividades específicas a través del juego terapéutico en un grupo de doce niños de tercero de preescolar para mejorar y fortalecer su psicomotricidad. A la vez, se propone el uso de un Centro Estimulación Multisensorial donde los niños disfrutan y controlan una variedad de experiencias sensoriales. Cabe mencionar que previo al inicio de las sesiones prácticas dentro del CEMS con los niños, se realizó un diagnóstico previo en el Jardín de Niños con sesenta niños los cuales presentaban ciertos parámetros de deficiencia en su desarrollo motor, los cuales fueron referidos por sus maestras. Una vez que se valoró a dicha población y tomando en cuenta los resultados, los criterios de inclusión y de exclusión; se tomó la determinación de que solo se trabajaría con doce niños, los cuales presentaban cierta deficiencia en su psicomotricidad, y es por ello que dicho diagnóstico nos permitió conocer con la población con la que se trabajaría. A continuación, se presenta evidencia de la primera evaluación realizada en el Jardin de Niños.

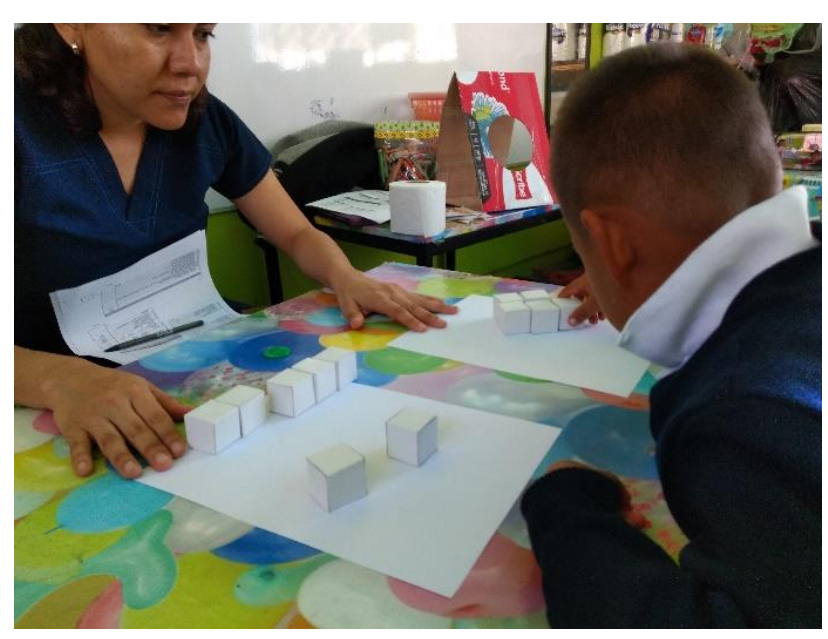

Figura 1 Diagnóstico inicial

Fuente. Elaboración propia 
A continuación, se muestra la estructura que se llevó a cabo en un plan de trabajo, por medio de actividades desarrolladas de la siguiente manera:

Dentro del desarrollo del trabajo práctico referente al uso del CEMS por medio del juego terapéutico con actividades específicas para favorecer la psicomotricidad en doce niños de preescolar, se plantea un plan de trabajo, el cual tiene la finalidad de brindar un seguimiento congruente por medio de una guía de actividades, divididas en diez sesiones de una duración cada sesión de una hora cada una, se realizaban dos días a la semana y dentro del CEMS. Se iniciaba trabajando con una relajación, después con la realización de dos a tres actividades basadas en el juego terapéutico y la psicomotricidad (la lateralidad y la praxia global) por último, se cerraba la sesión con un recordatorio de las actividades realizadas con los niños para que estas a la vez las reforzarán en casa.

A continuación, se muestra una de las sesiones trabajadas dentro del CEMS. Esta corresponde al inicio de dicha sesión y se desarrolla la etapa de relajación.

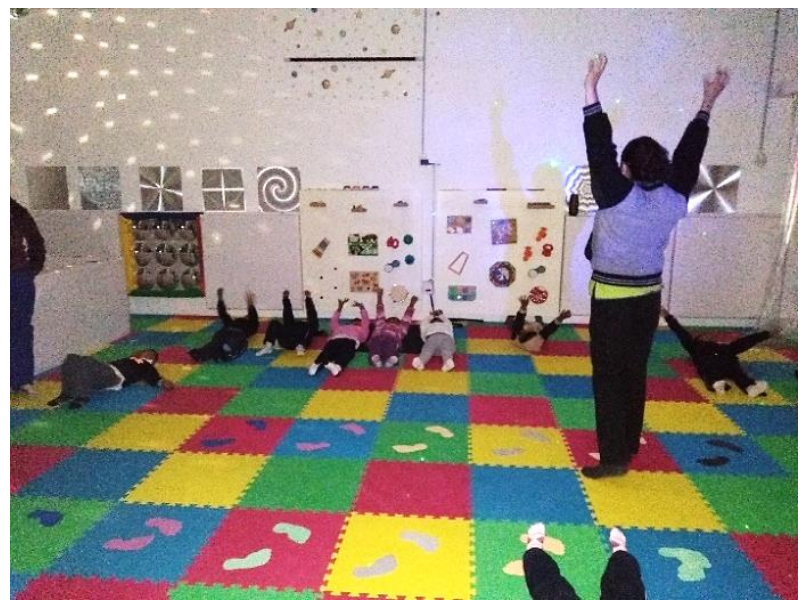

Figura 2 Relajación

Fuente. Elaboración propia.

Después se prosigue con una de las actividades de psicomotricidad basadas en el juego terapéutico, específicamente se trabajó la lateralidad de miembros superiores.

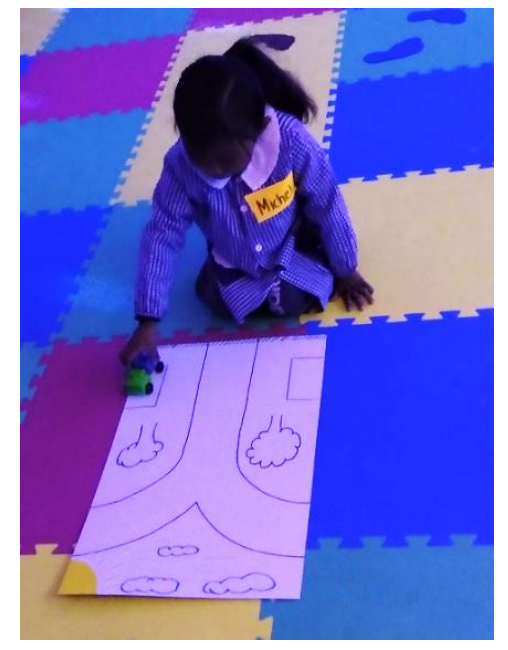

Figura 3 Actividad de lateralidad

Fuente. Elaboración propia

La siguiente figura hace referencia al trabajo de la lateralidad de miembros inferiores.

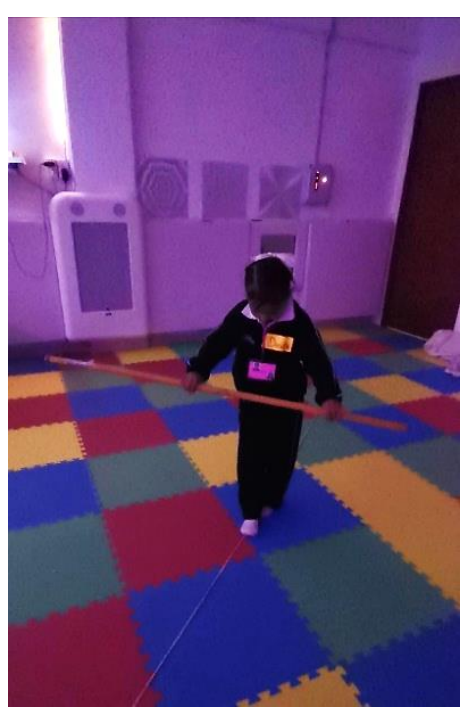

Figura 4 Actividad de lateralidad

Fuente. Elaboración propia

Por último, la sesión se cierra con un recordatorio de las actividades realizadas en el día y se acompaña de una actividad lúdica.

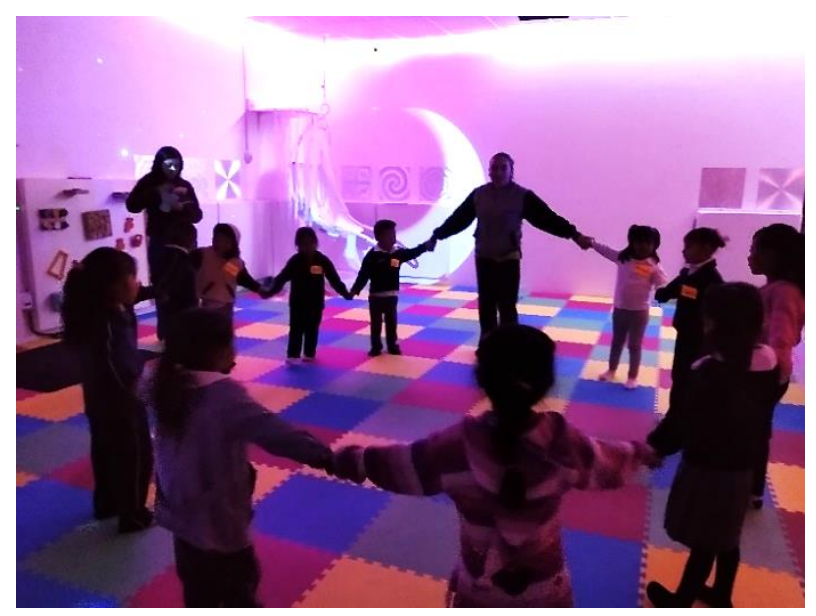

Figura 5 Actividad de cierre de sesión

Fuente. Elaboración propia 


\section{Resultados}

Para obtener resultados cuantitativos con respecto a las actividades de psicomotricidad, se realizaron dos evaluaciones; una previa la cual se desarrolló en el Jardín de Niños para evaluar su nivel de maduración en el aspecto motriz por medio de la Escala de McCarthy de aptitudes y psicomotricidad para niños (MSCA) y una segunda después de la intervención de las diez sesiones con actividades lúdicas utilizando la misma escala de evaluación; para conocer el impacto que tiene la psicomotricidad siendo utilizada como herramienta en el juego terapéutico dentro de un CEMS.

Cada sesión contó con diferentes actividades y estas a su vez estaban basadas a los resultados correspondientes al diagnóstico inicial arrojados por la Escala MSCA.

Por lo tanto, en la siguiente tabla 1 se muestran los resultados iniciales correspondiente al diagnóstico que se aplicaron a doce niños, donde se puede observar que existe una falta de maduración en su psicomotricidad.

Escala de McCarthy de aptitudes y psicomotricidad para niños
(MSCA)
\begin{tabular}{|r|r|r|r|r|}
\hline \multicolumn{5}{|c|}{ Diagnóstico } \\
\hline \multicolumn{5}{|c|}{ Puntuación máxima } \\
\hline \multicolumn{2}{|r|}{$\begin{array}{l}\text { Orientación } \\
\text { izq-der }\end{array}$} & $\begin{array}{l}\text { Coordinación } \\
\text { de piernas }\end{array}$ & $\begin{array}{l}\text { Coordinación } \\
\text { de brazos }\end{array}$ & Imitación \\
\hline 1 & 6 & 10 & 2 & 2 \\
\hline 2 & 4 & 9 & 1 & 3 \\
\hline 3 & 4 & 8 & 1 & 4 \\
\hline 4 & 3 & 9 & 0 & 2 \\
\hline 5 & 3 & 10 & 0 & 3 \\
\hline 6 & 6 & 11 & 1 & 3 \\
\hline 7 & 2 & 7 & 1 & 2 \\
\hline 8 & 2 & 8 & 0 & 4 \\
\hline 9 & 7 & 8 & 2 & 2 \\
\hline 10 & 1 & 9 & 3 & 3 \\
\hline 11 & 3 & 8 & 5 & 2 \\
\hline 12 & 4 & 9 & 4 & 3 \\
\hline
\end{tabular}

Tabla 1 Evaluación diagnóstica Fuente: Elaboración propia

Por último, los resultados obtenidos después de la aplicación de actividades basadas en el juego terapéutico, se observa un impacto positivo en su nivel de psicomotricidad de cada niño.

\begin{tabular}{|c|c|c|c|c|}
\hline \multicolumn{5}{|c|}{ Escala de McCarthy de aptitudes y psicomotricidad para niños } \\
\hline Despt & de la & de & & \\
\hline \multicolumn{5}{|c|}{ Puntuación máxima } \\
\hline & 9 & 13 & 7 & 4 \\
\hline Niño & Orientación & Coordinación & $\begin{array}{l}\text { Coordinación } \\
\text { de brazos }\end{array}$ & Imitación \\
\hline 1 & 8 & 13 & 5 & 4 \\
\hline 2 & 8 & 11 & 4 & 4 \\
\hline 3 & 7 & 12 & 5 & 4 \\
\hline 4 & 8 & 12 & 4 & 4 \\
\hline 5 & 7 & 13 & 5 & 4 \\
\hline 6 & 9 & 13 & 6 & 4 \\
\hline 7 & 7 & 10 & 5 & 3 \\
\hline 8 & 7 & 11 & 4 & 4 \\
\hline 9 & 9 & 12 & 6 & 4 \\
\hline 10 & 7 & 12 & 6 & 4 \\
\hline 11 & 8 & 11 & 7 & 3 \\
\hline 12 & 9 & 12 & 6 & 4 \\
\hline
\end{tabular}

Tabla 2 Evaluación posterior a las actividades Fuente: Elaboración propia

\section{Conclusiones}

Esta investigación permite demostrar como el trabajar por medio de actividades específicas y el juego terapéutico fortalece la psicomotricidad. Además, con el apoyo del CEMS dichas actividades se adecuaron, lo que generó otras formas de planear y organizar. Por último, aunque no fue parte de los objetivos a alcanzar se observó que en los niños hubo un aumento en su concentración, centraron mejor su atención, su estado de alerta, el despertar recuerdos, mejorar la movilidad, la creatividad, las relaciones sociales y las comunicaciones en general el conocimiento del mundo circundante; esto debido a los comentarios realizados por sus maestras a cargo y los padres de familia.

\section{Referencias}

Abarca Mora Sonia, "Psicología del niño en edad escolar", Costa Rica: EUNED. pp.64-69. Dirección internet:https://books.google.es/books?id=PjnW BndwNJ8C\&pg=PA61\&dq=piaget,+etapas+de + desarrollo+del+ni\%C3\%B1o\&hl=es\&sa $=X \&$ e $\mathrm{i}=\mathrm{y} 5 \mathrm{DuVOmuAeHas} A \mathrm{SIm} Y \mathrm{DAB} w \& v e d=0 \mathrm{CC}$ AQ6AEwAA\#v=onepage $\& q=$ piaget $\% 2 \mathrm{C} \% 20$ et apas\%20de $\% 20$ desarrollo\%20del\%20ni\%C3\% $\mathrm{B} 1 \mathrm{o} \& \mathrm{f}=$ false, 2007.

Arnaiz, P., Rabadán, M., Vives, I. (2008). La psicomotricidad en la escuela: una práctica preventiva y educativa. España: Aljibe, Ediciones. 
Berruezo, P. (2008). El contenido de la Psicomotricidad. Reflexiones para la delimitación de su ámbito teórico y práctico. Recuperado de file://C:/Users/KCAA/Downloads/Dialnet-

ElContenidoDeLaPsicomotricidad-2707331.pdf

Campo, L., Jiménez, P., Maestre, K., Paredes, N. (2011). Características del desarrollo motor en niños de 3 a 7 años de la Ciudad de Barranquilla. Psicogente, 14(25), 76-89.

Carbajo, M. (2014). La sala de estimulación multisensorial. Recuperado de https://uvadoc.uva.es/bitstream/handle/10324/1 6701/Tabanque-2014-27-

LaSalaDeEstimulacionMultisensorial.pdf?seque nce $=1$

Challis, B., Kang, A., Rimmer, R., y Hildred, M. (2017). Enhancing the Multisensory Environment with Adaptive Game Audio Techniques. A.L. Brooks and E. Brooks (Eds.): ArtsIT/DLI 2016, LNICST, 154-161. doi: 10.1007/978-3-319-55834-9_18

Chinome, J., Rodríguez, L., Parra, J. (2017). Implementación y evaluación de un programa de estimulación cognitiva en preescolares rurales. Psicología desde el Caribe, 34 (3), 184203.

Fábry, Z. (2019). Psychomotor therapy in the context of multisensory environment. Journal of Exceptional People, 1(14), 7-14.

Ibáñez, P., Mudarra, J., Alfonso, C. (2004). La estimulación psicomotriz en la infancia a través del método estitsológico multisensorial de atención temprana. Recuperado de https://www.redalyc.org/pdf/706/70600706.pdf

Mas, L. (2013). La Práctica de la Psicomotricidad Educativa y Preventiva. Recuperado de https://dspace.uib.es/xmlui/bitstream/handle/11 201/145367/Mas_Trujillo_Laura.pdf?sequence =1\&is Allowed=y 